\title{
带有扩散项的 Brusselator 模型的 近似解析解
}

\author{
张 锁 春 \\ （中国科学院应用数学研究所，北京）
}

带有扩散项的 Brusselator 模型所对应的速率方程是非线性抛物型的偏微分方程组. Prigogine 学派付对它研究了情况 (I)：A和 B 都是均匀的; 情况 (II)：A是非均匀, B 是均匀 的. 在研究中采用的基本方法是线性化加上小参数, 在分歧点附近作展开, 得到解的近似解析 表达式, 并由数值模拟作检证. 其方法对情况 (III)：A和 B 都是非均匀的研究却遇到了困 难, 至今未见这方面的结果. 本文利用 “计算机近似解法”[2] 给出了情况 (III) 的近似解析 解.

\section{一、方程和边界条件}

带有扩散项的 Brusselator 模型在非均匀介质中所对应的一维空间的速率方程是

$$
\begin{aligned}
& \left\{\begin{array}{l}
\partial A / \partial t=-A+D_{A} \partial^{2} A / \partial r^{2}, \\
\partial X / \partial t=A-(B+1) X+X^{2} Y+D_{X} \partial^{2} X / \partial r^{2}, \\
\partial Y / \partial t=B X-X^{2} Y+D_{Y} \partial^{2} Y / \partial r^{2}, \\
\partial B / \partial t=-B X+D_{B} \partial^{2} B / \partial r^{2},
\end{array}\right. \\
& \partial D / \partial t=B X+D_{D} \partial^{2} D / \partial r^{2}, \\
& \partial E / \partial t=X+D_{E} \partial^{2} E / \partial r^{2},
\end{aligned}
$$

其中 $D_{i}(i-A, X, Y, B, D, E)$ 是扩散系数, $0 \leqslant r \leqslant 1$.

对于 $D 、 E$ 的方程, 在 $B 、 X 、 Y$ 已解出的情况下, 它们只不过是非齐次的线性方程, 而且 不与 $X 、 Y$ 的方程发生联系,故可忽略不予考虑.

边界条件是 Dirichlet 条件:

$$
\left\{\begin{array}{l}
A(0, t)-A(1, t)=\bar{A}, \\
B(0, t)=B(1, t)=\bar{B}, \\
X(0, t)-X(1, t)-\bar{A}, \quad(t \geqslant 0) \\
Y(0, t)-Y(1, t)=\bar{B} / \bar{A},
\end{array}\right.
$$

其中 $\bar{A}, \bar{B}$ 是给定的常数. 初始条件可任意给定.

\section{二、数值模拟结果}

我们利用 Herschkowitz-Kaufman ${ }^{[3]}$ 等人在研究情况（II）时的两个基本模型参数:

本文 1981 年 5 月 20 日收到. 
模型 I: $\bar{A}-14, \bar{B}=77, D_{A}=0.197, D_{\mathrm{X}}=0.00105, D_{\mathrm{Y}}=0.00066$.

模型 II: $\bar{A}=14, \vec{B}=26, D_{A}=0.197, D_{X}=0.00105, D_{Y}=0.00525$.

作为我们对情况 (III) 进行数值模拟的出发点. 对模型 I 在 $D_{B}$ 较大变化范围内取不同的值 进行计算. 对模型 II 仅取 $D_{B}=0.197$ 进行计算.

计算结果见表 $1-2$. 由于定常态解具有空间对称性,故只列出一半的数据. 由于篇幅所 限, 仅列出 $\boldsymbol{X}$ 的数据.

表 1 模型 $\mathrm{I}$ 参数下 $\boldsymbol{x}$ 的定常态解

\begin{tabular}{c|c|c|c|c|c|c|c|c}
\hline & & & & \\
\hline & 0 & 0.026 & 0.051 & 0.103 & 0.154 & 0.231 & 0.308 & 0.487 \\
\hline$D_{B}$ & & & & & & & & \\
\hline 0 & 14.00 & 14.99 & 13.58 & 11.96 & 10.95 & 9.80 & 9.07 & 8.28 \\
\hline $0.197 \times 10^{-3}$ & 14.00 & 14.95 & 13.58 & 11.95 & 10.93 & 9.83 & 9.05 & 8.26 \\
\hline $0.197 \times 10^{-2}$ & 14.00 & 14.66 & 13.59 & 11.97 & 10.94 & 9.83 & 9.00 & 8.26 \\
\hline $0.197 \times 10^{-1}$ & 14.00 & 13.91 & 13.31 & 12.04 & 11.02 & 9.89 & 9.10 & 8.33 \\
\hline 0.197 & 14.00 & 13.48 & 12.92 & 11.88 & 10.98 & 9.91 & 9.14 & 8.38 \\
\hline $0.197 \times 2$ & 14.00 & 13.44 & 12.87 & 11.83 & 10.93 & 9.85 & 9.06 & 8.28 \\
\hline $0.197 \times 4$ & 14.00 & 13.42 & 12.85 & 11.81 & 10.91 & 9.84 & 9.06 & 8.27 \\
\hline $0.197 \times 8$ & 14.00 & 13.42 & 12.85 & 11.82 & 10.92 & 9.84 & 9.05 & 8.24 \\
\hline $0.197 \times 10$ & 14.00 & 13.41 & 2.84 & 11.80 & 10.90 & 9.82 & 9.04 & 8.26 \\
\hline $0.197 \times 14$ & 14.00 & 13.41 & 12.84 & 11.79 & 10.90 & 9.82 & 9.04 & 8.25 \\
\hline
\end{tabular}

表 2 模型 II 参数下的定常态解 $\left(D_{B}=0.197\right)$

\begin{tabular}{c|c|c|c|c|c|c|c|c|c|c}
\hline$r$ & 0 & 0.026 & 0.051 & 0.077 & 0.103 & 0.154 & 0.231 & 0.333 & 0.487 & 0.500 \\
\hline$B$ & 26.00 & 21.19 & 15.73 & 14.27 & 11.79 & 8.15 & 4.84 & 2.63 & 1.60 & 1.59 \\
\hline$X$ & 14.00 & 13.65 & 12.81 & 12.53 & 11.99 & 11.04 & 9.91 & 8.90 & 8.30 & 8.30 \\
\hline$Y$ & 1.86 & 1.56 & 1.23 & 1.14 & 0.98 & 0.74 & 0.49 & 0.30 & 0.19 & 0.19 \\
\hline
\end{tabular}

计算结果表明:

1. 取定一组参数后, 不管初始条件如何选取,方程组 (1.1) 在边界条件 (1.2) 下的解都趋 向于一个定常态解。

2. 当 $D_{B}$ 比较小时,约与 $D_{X} 、 D_{Y}$ 同数至级,存在边界效应.

3. 当 $D_{B}$ 充分大时, 约比 $D_{A}$ 大一个数量级,定常态解与 $D_{B}$ 选择无关,这些解趋向于同一 个稳定的分布.

4. 在边界条件 (1.2)下, 方程组 (1.1) 的解随时间演变的过程, 可参见图 1, 它清楚地反映 了反应扩散过程。 


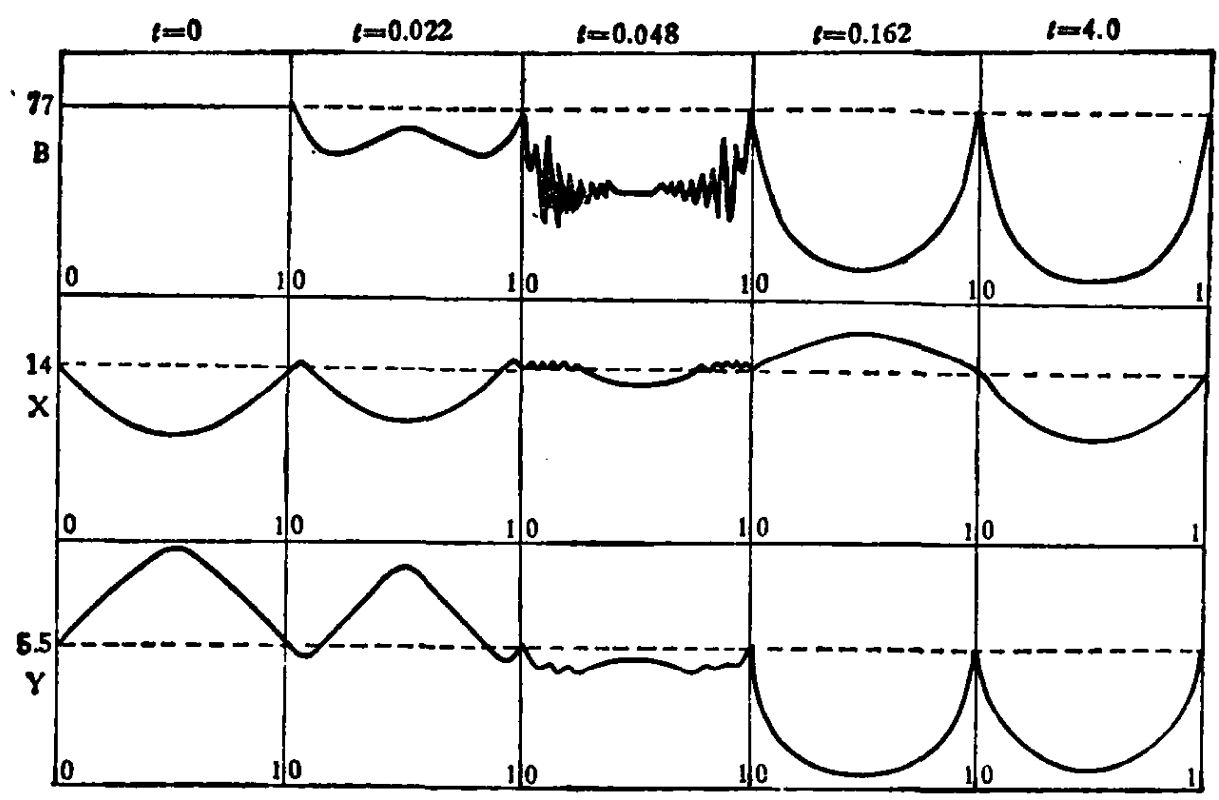

图 $1 B 、 X 、 Y$ 随时间 2 的演变图

参数同模型 I. $D_{B}=0.197$

\section{三、近似解析解}

数值结果使得我们在构造方程组 (1.1) 在边界条件 (1.2) 下的近似解析解时能够选取适当 的解析形式. 当 $t \rightarrow \infty$ 时, 定常态解的近似解析表达式是

$$
\left\{\begin{array}{l}
A(r)=\bar{A} \operatorname{sech}\left(1 /\left(2 \sqrt{D_{A}}\right)\right) \cdot \cosh \left(\left(r-\frac{1}{2}\right) / \sqrt{D_{A}}\right), \\
B(r)=\bar{B} \operatorname{sech}\left(\sqrt{A(r)} /\left(2 \sqrt{D_{B}}\right)\right) \cdot \cosh \left(\left(r-\frac{1}{2}\right) \cdot \sqrt{A(r)} / \sqrt{D_{B}}\right), \\
X(r)=A(r), \\
Y(r)=B(r) / A(r) .
\end{array}\right.
$$

公式(3.1)的近似程度见表 3、4.

表 3 模型 I 数下 $x$ 的定常态解的结果比较

\begin{tabular}{|c|c|c|c|c|c|c|c|c|}
\hline$D_{B}$ & & 0.026 & 0.051 & 0.103 & 0.154 & 0.231 & 0.308 & 0.487 \\
\hline \multirow{3}{*}{$0.197 \times 14$} & 公式结果 & 13.37 & 12.78 & 11.73 & 10.84 & 9.77 & 9.00 & 8.22 \\
\hline & 数值结果 & 13.41 & 12.84 & 11.79 & 10.90 & 9.82 & 9.04 & 8.25 \\
\hline & 相对误差 & $3 \%$ & $5 \%$ & $5 \%$ & $5.5 \%$ & $5 \%$ & $4 \%$ & $3.6 \%$ \\
\hline \multirow{3}{*}{$0.197 \times 10^{-1}$} & 公式结果 & 13.37 & 12.78 & 11.73 & 10.84 & 9.77 & 9.00 & 8.22 \\
\hline & 数值结果 & 13.91 & 13.31 & 12.04 & 11.02 & 9.89 & 9.10 & 8.33 \\
\hline & 相对误差 & $3.8 \%$ & $4 \%$ & $2.6 \%$ & $1.6 \%$ & $1 \%$ & $1 \%$ & $1 \%$ \\
\hline
\end{tabular}


表 4 模型 I 参数下 $Y$ 的定常态解的结果比较

\begin{tabular}{|c|c|c|c|c|c|c|c|c|}
\hline$D_{B}$ & 类型 & 0.026 & 0.051 & 0.103 & 0.154 & 0.231 & 0.308 & 0.487 \\
\hline \multirow{3}{*}{$0.197 \times 14$} & 公式结果 & 5.51 & 5.53 & 5.63 & 5.77 & 6.04 & 6.32 & 6.71 \\
\hline & 数值结果 & 5.54 & 5.58 & 5.70 & 5.83 & 6.04 & 6.16 & 6.46 \\
\hline & 相对误差 & $5 \%$ & $9 \%$ & $1 \%$ & $1 \%$ & 0 & $1.8 \%$ & $3.9 \%$ \\
\hline \multirow{3}{*}{$0.197 \times 10^{-1}$} & 公式结果 & 2.95 & 1.63 & 0.54 & 0.19 & 0.046 & 0.0076 & 0.00071 \\
\hline & 数值结果 & 2.86 & 1.56 & 0.49 & 0.16 & 0.033 & 0.0068 & 0.00030 \\
\hline & 相对误差 & $3 \%$ & $4.5 \%$ & $10 \%$ & $18.8 \%$ & $39 \%$ & $11.8 \%$ & $137 \%$ \\
\hline
\end{tabular}

总的说来,近似公式与数值结果符合得很好. 在某些点上相对误差显得较大,但其绝对误 差是很小的.

致谢: 作者十分感谢秦元勋教授的指导.

\section{$\Rightarrow$ 文}

[1] Nicolis, G. \& Prigogine, I., Self-Organization in Nonequilibrium Systems, John Wiley \& Sons. Inc., 1977.

[2] 张锁春, 科学通报, 26(1981), 7: 446 .

[ 3 ] Herschkowitz-Kaufman, M. \& Platten, J., Bull. Acad. Roy. Belg. Cl. Sci., 57 (1971), 26. 